TITLE:

\title{
AFM analysis of changes in nucleosome wrapping induced by DNA epigenetic modification
}

\section{$\operatorname{AUTHOR}(S)$ :}

Kizaki, Seiichiro; Suzuki, Yuki; Takenaka, Tomohiro; Endo, Masayuki; Sugiyama, Hiroshi

\section{CITATION:}

Kizaki, Seiichiro ... [et al]. AFM analysis of changes in nucleosome wrapping induced by DNA epigenetic modification. Biomaterials Science 2014, 2(10): 1399-1403

\section{ISSUE DATE:}

2014-06-13

URL:

http://hdl.handle.net/2433/198840

\section{RIGHT:}

This journal is (C) The Royal Society of Chemistry 2014.; この論文は出版 社版でありません。引用の際には出版社版をご確認ご利用ください。; This is not the published version. Please cite only the published version. 


\section{ARTICLE}

Cite this: DOI: $10.1039 /$ xoxxooooox

Received ooth January 2012

Accepted ooth January 2012

DOI: $10.1039 / \times 0 x \times 00000 x$

www.rsc.org/

\section{AFM analysis of changes in nucleosome wrapping induced by DNA epigenetic modification}

\author{
Seiichiro Kizaki, ${ }^{a}$ Yuki Suzuki, ${ }^{a}$ Tomohiro Takenaka, ${ }^{a}$ Masayuki Endo ${ }^{b}$ and \\ Hiroshi Sugiyama ${ }^{\mathrm{a}, \mathrm{b}}$
}

The wrapping and unwrapping of the nucleosome, which is a fundamental packing unit of chromatin, are tied to the regulation of gene expression. The accessibility of DNA within nucleosomes is controlled not only by chromatin-remodeling molecules, but also by chemical modifications of histones and DNA. Understanding the structural changes of a nucleosome during epigenetic modifications is a key to the unraveling of the mechanisms of gene regulation. Here, we reconstituted nucleosomes using methylcytosine- or hydroxymethylcytosine-substituted DNA, and analyzed their morphological features by atomic force microscopy (AFM). Our results indicate that cytosine methylation induces overwrapping of the DNA around the histone octamer, whereas cytosine hydroxymethylation has a lesser effect on the overwrapping of the DNA. These results suggest that two types of DNA modification yield different wrapping states of nucleosomes, which may contribute to the compaction and relaxation of chromatin structure.

\section{Introduction}

Eukaryotic genomic DNA interacts with various proteins and is folded into chromatin fibers. The most fundamental unit of chromatin is the nucleosome, which is composed of $\sim 147$ bp DNA that wraps in $\sim 1.75$ turns around a histone octamer that contains two copies of $\mathrm{H} 2 \mathrm{~A}, \mathrm{H} 2 \mathrm{~B}, \mathrm{H} 3$, and H4. ${ }^{1,2}$ The nucleosome represents a significant barrier for DNA-binding regulatory proteins that control the process of gene expression. Therefore, changes in nucleosome structure are closely related to gene regulation.

The key mechanisms that modulate nucleosome properties are enzyme-mediated modifications of histones and DNA. Among these epigenetic modifications, the methylation of cytosine in DNA is essential in genomic imprinting, retrotransposon silencing, and $\mathrm{X}$ chromosome inactivation. ${ }^{3-5}$ In mammalian cells, DNA methyltransferase (DNMT) transfers the methyl group of $S$ adenosylmethionine (SAM) to cytosine in $\mathrm{CpG}$ dinucleotides. In comparison with other epigenetic modifications, the methylation of cytosine is a relatively stable modification that is usually maintained throughout the cell cycle. ${ }^{6,7}$ However, in specific developmental stages, such as developing primordial germ cells (PGCs), methylation of cytosine is rapidly removed. ${ }^{8,9}$ Although the molecular mechanism underlying this active demethylation remains unclear, recent studies revealed that Tet-family proteins have an activity to convert methylcytosine (mC) to hydroxymethylcytosine (hmC), and further to formylcytosine (fC) and carboxylcytosine (caC) (Fig. 1). ${ }^{10-12}$ These oxidized derivatives of $\mathrm{mC}$ are now considered intermediates of the active demethylation pathway. ${ }^{13}$
To understand the structural changes of nucleosomes that occur upon DNA methylation and demethylation, it will be necessary to image the nucleosome on modified DNA strands. In the studies communicated here, we have taken advantage of the ability of atomic force microscopy (AFM) to provide images of DNA-protein complexes in solution to investigate the structure of nucleosomes that were reconstituted on unmethylated, methylated, and hydroxymethylated DNA. Previously the group of Lyubchenko used AFM imaging to directly visualize the dynamics of nucleosomes ${ }^{14}$ and they revealed that histone $\mathrm{H} 4$ biotinylation significantly increases the length of DNA wrapped around the histone octamer. ${ }^{15}$ In the present study, we showed that DNA epigenetic modification changes the extent of wrapping of DNA around the histone octamer.

\section{Experimental}

\section{DNAs and histones}

The 381 bp DNA fragment containing 601 positioning sequence was amplified by PCR using forward 5'-

TAATACGACTCACTATAGG-3' and reverse 5'-

ATTTAGGTGACACTATAGAATAC-3' primers from pGEM3Z$601{ }^{16}$ After the reaction, the amplified DNA was purified using GenElute PCR Clean-Up Kit (Sigma-Aldrich, St. Louis, MO, USA). Methylcytosine- and hydroxymethylcytosine-substituded DNAs were prepared by conducting the PCR with 5-Methylcytosine dNTP Mix (Zymo Research, Irvine, CA, USA) and 5-

Hydroxymethylcytosine dNTP Mix (Zymo Research), respectively. 
HeLa core histone was purchased from Active Motif (Carlsbad, CA, USA).

(a)

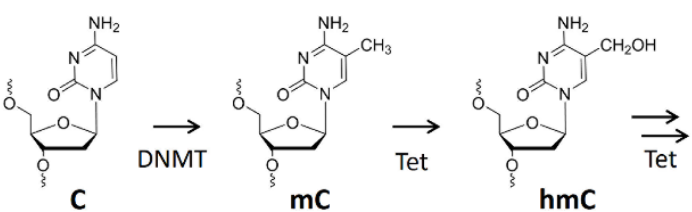

(b) Plasmid containing 601 sequence

$$
\begin{array}{rlrl}
\text { (pGEM3Z-601) } & \mathrm{C}^{*} & =\text { cytosine }(\mathbf{C}) \\
\mathrm{PCR} \_\mathrm{dATP}, \mathrm{dGTP}, \mathrm{TTP}, \mathrm{dC} * \mathrm{TP} & =5 \text {-methylcytosine }(\mathrm{mC}) \\
& =5 \text {-hydroxymethylcytosine }(\mathbf{h m C})
\end{array}
$$

$381 \mathrm{bp}(\sim 130 \mathrm{~nm})$

$137 \mathrm{bp}(\sim 46.5 \mathrm{~nm}) \quad 601$ sequence $(146 \mathrm{bp}) \quad 98 \mathrm{bp}(\sim 33 \mathrm{~nm})$

reconstitution $\square$ histone octamer

nucleosome

(c)
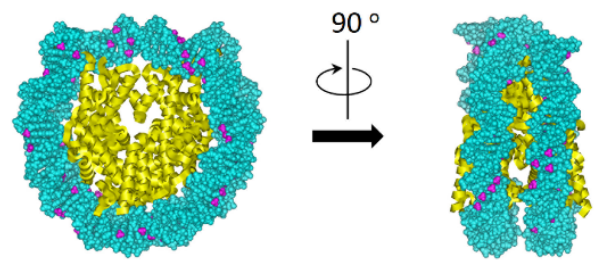

Fig. 1 Study on the effect of cytosine modification on nucleosome formation. (a) Cytosine modification for the epigenetic gene regulation. (b) Experimental scheme for the nucleosome reconstitution using a PCR amplified reconstitution sequence containing modified cytosine. The location of the 601 sequence along a $381 \mathrm{bp}$ fragment is shown. (c) The cytosinemethylated nucleosome model was constructed using the crystal structure of Xenopus laevis nucleosome core particle (Protein Data Bank Code: 3LZ0). Methyl groups of all cytosines are colored in purple.

\section{Nucleosome reconstitution}

Nucleosomes were reconstituted as described previously. ${ }^{17,18}$ Briefly, equal amounts $(0.5 \mathrm{mg})$ of the purified DNA and the histone octamer were mixed in Hi-buffer [10 mM Tris- $\mathrm{HCl}$ (pH 7.5), $2 \mathrm{M} \mathrm{NaCl}, 1$ mM EDTA, $0.05 \% \mathrm{NP}-40$, and $5 \mathrm{mM} \beta$-mercaptoethanol], and placed in a dialysis tube (total volume $50 \mathrm{~mL}$ ). The dialysis was started in $150 \mathrm{~mL}$ of Hi-buffer with stirring at $4{ }^{\circ} \mathrm{C}$. Lo-buffer [10 $\mathrm{mM}$ Tris- $\mathrm{HCl}$ (pH 7.5), $1 \mathrm{mM}$ EDTA, $0.05 \% \mathrm{NP}-40$, and $5 \mathrm{mM} \beta$ mercaptoethanol] was added to the dialysis buffer at the rate of 0.46 $\mathrm{mL} / \mathrm{min}$, and the dialysis buffer was pumped out at the same rate with a peristaltic pump so that the final dialysis buffer contained 50 $\mathrm{mM} \mathrm{NaCl}$ after $20 \mathrm{~h}$. The sample was collected from the dialysis tube and stored at $4{ }^{\circ} \mathrm{C}$ until use.

\section{AFM imaging}

The reconstituted nucleosome was diluted to a concentration of 0.5 $\mathrm{ng} / \mu \mathrm{L}$ in a buffer containing $20 \mathrm{mM}$ Tris- $\mathrm{HCl}(\mathrm{pH} 7.5), 10 \mathrm{mM}$ $\mathrm{MgCl}_{2}$, and $1 \mathrm{mM}$ EDTA, and $3 \mu \mathrm{L}$ of the sample was immediately deposited onto freshly cleaved mica discs $(\varphi 1.5 \mathrm{~mm})$ pretreated with
0.1\% (3-Aminopropyl)triethoxysilane (APTES). After $1 \mathrm{~min}$ incubation, the sample was rinsed with $2 \times 10 \mu \mathrm{L}$ washes of the buffer and then imaged in the same buffer without the drying step. The AFM experiments were performed using a high-speed AFM (Nano Live Vision, RIBM, Tsukuba, Japan). The sample was imaged in buffer solution at ambient temperature with a small cantilever of dimensions $\mathrm{L} \times \mathrm{W} \times \mathrm{H}=10 \times 2 \times 0.1 \mu \mathrm{m}^{3}$ (BLAC10EGS, Olympus, Tokyo, Japan). These cantilevers had a spring constant of $0.1-0.2 \mathrm{~N} / \mathrm{m}$ with a resonant frequency in water of $400-1000 \mathrm{kHz}$ and $320 \times 240$ pixel images were obtained at the scan rate of $0.2 \mathrm{fps}$. Individual images were imported into ImageJ (http://rsb.info.nih.gov/ij/) and analyzed. The length of wrapped DNA was calculated by subtracting the sum of the measured lengths of both DNA arms from the theoretical length of template DNA.

\section{Results and Discussion}

The use of reconstituted nucleosomal systems that contain a set of nucleosome-positioning signals has been a powerful approach to the analysis of the structure-function relationship of nucleosomes. ${ }^{19,20}$ We used the fragment of 381 bp carrying the 146 bp nucleosomepositioning 601 sequence flanked with two DNA arms of different lengths, 137 and 98 bp (Fig. 1b). AFM images in a liquid of the reconstituted nucleosomes depict a typical morphology for the mononucleosome, i.e., a bright particle with the two DNA arms at both sides (Fig. 2a). Note that the prepared sample was not treated with any crosslinkers, such as glutaraldehyde. The mean lengths of the longer and shorter arms $( \pm \mathrm{SD})$ were $50.8 \pm 9.3 \mathrm{~nm}$ and $39.3 \pm$ $7.7 \mathrm{~nm}$, respectively (Fig. 2b and c), which was close to the expect tengths of $46.6 \mathrm{~nm}$ for the longer arm and $33.3 \mathrm{~nm}$ for the shorter arm based on a nanometer-to-base-pair conversion factor of 0.34 $\mathrm{nm} / \mathrm{bp}$. The broad range of the length distribution may reflect the dynamic feature of nucleosomes, ${ }^{21-24}$ thus allowing varying extents of DNA wrapping, ranging from $\sim 1$ to $\sim 1.75$ turns. ${ }^{14}$ (a)

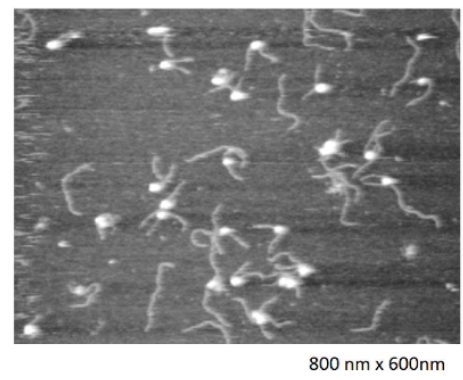

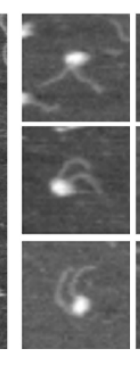

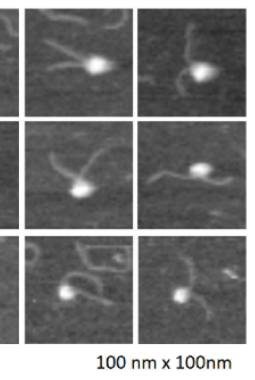

(b)

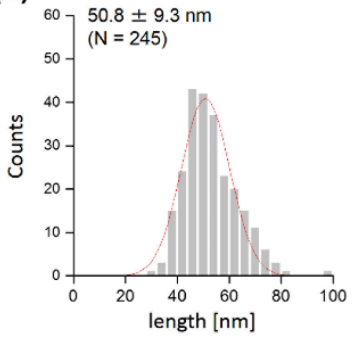

(c)

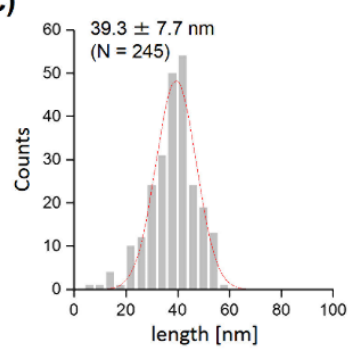

Fig. 2 AFM imaging of the reconstituted nucleosome. (a) AFM images of nucleosomes that were reconstituted on unmethylated DNA. Lower magnification: image size, $800 \mathrm{~nm} \times 600 \mathrm{~nm}$; higher magnification: image 
size, $100 \mathrm{~nm} \times 100 \mathrm{~nm}$. (b) Histogram of the measured contour lengths of the longer arms. (c) Histogram of the measured contour lengths of the shorter arms. A Gaussian-fitted curve is overlaid on each histogram.

We were interested in determining whether the structure of the nucleosome changes after the methylation of the template DNA (Fig. 3a). To assess this, we reconstituted nucleosomes using an $\mathrm{mC}$ substituted DNA template and compared their features with those of unmethylated nucleosomes. Intriguingly, in AFM images (Fig. 3b), the assembled methylated nucleosomes were more compact than were the unmethylated ones. The analysis of the contour length of the two DNA arms provided quantitative information about this observation. As shown in Fig. 3c and d, the frequency distribution of the longer and shorter arms of the methylated nucleosome showed a peak at $45.5 \pm 8.3 \mathrm{~nm}$ and $33.6 \pm 8.7 \mathrm{~nm}$, respectively. These values were smaller than the values obtained for the unmethylated nucleosomes (Fig. 2b), which suggests that cytosine methylation induces overwrapping of DNA around the histone octamer in a nucleosome. To assess the degree of nucleosome wrapping, we calculated the length of wrapped DNA for all nucleosomes, as described in the Methods section. Fig. 4a and $\mathrm{b}$ shows the histograms of this value for the unmethylated nucleosomes and for the cytosine-methylated nucleosomes, respectively. Compared with unmethylated nucleosomes, an increase of $\sim 32 \mathrm{bp}$ in the average of wrapped DNA was observed in methylated nucleosomes. These results indicate that cytosine methylation changes nucleosome structure and is accompanied by overwrapping of DNA around the histone octamer. (a)

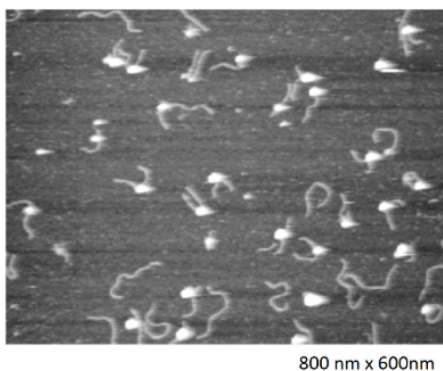

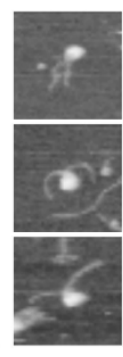

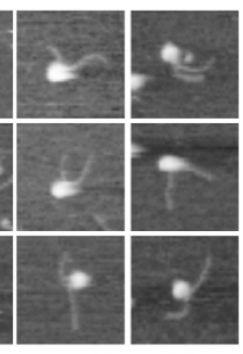

$100 \mathrm{~nm} \times 100 \mathrm{~nm}$ (b)

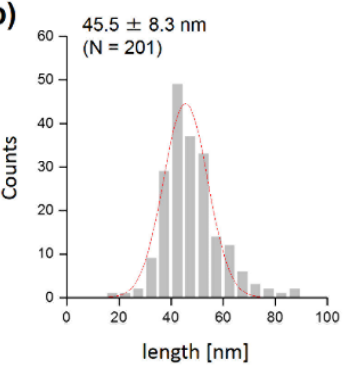

(c)

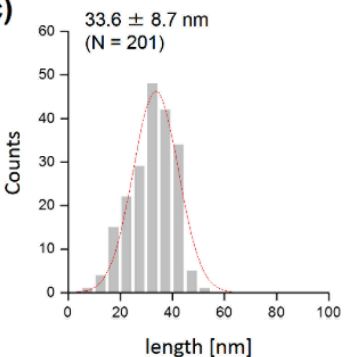

Fig. 3 AFM imaging of the cytosine-methylated nucleosome. (a) AFM images of nucleosomes that were reconstituted on methylated DNA. Lower magnification: image size, $800 \mathrm{~nm} \times 600 \mathrm{~nm}$; higher magnification: image size, $100 \mathrm{~nm} \times 100 \mathrm{~nm}$. (b) Histogram of the measured contour lengths of the longer arms. (c) Histogram of the measured contour lengths of the shorter arms. A Gaussian-fitted curve is overlaid on each histogram.
The effect of DNA methylation on the nucleosome structure has been studied extensively by targeting $\mathrm{CpG}$ dinucleotides. ${ }^{25,26}$ Our results from cytosine-methylated nucleosomes were in line with those of previous studies, in which $\mathrm{CpG}$ methylation was reported to induce the compaction and stabilization of the nucleosome by causing a tighter wrapping of DNA around the histone octamer. ${ }^{26}$ The studies of CpG-methylated nucleosome core particles suggest that the changes in the wrapping can be attributed to the reduced twist of DNA upon methylation. ${ }^{26}$ In our system, almost all cytosines in the $381 \mathrm{bp}$ are methylated, including the two flanking DNA arms. This highly methylated state of DNA might reduce its extent of twisting, causing a drastic increase in the average of wrapped DNA.
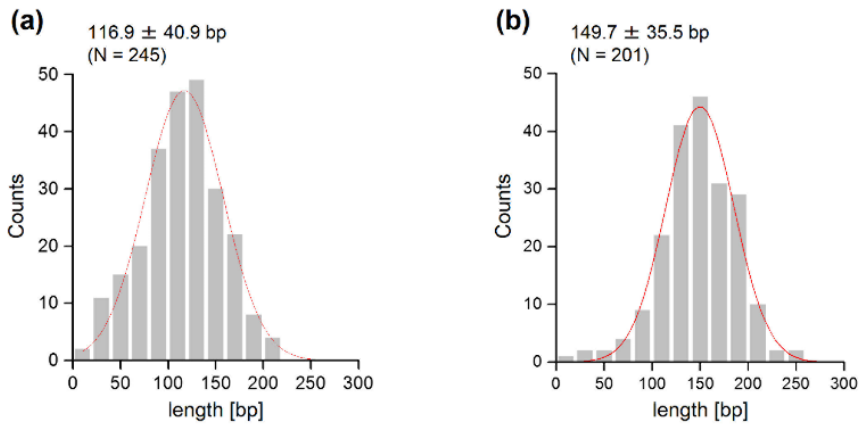

Fig. 4 Effect of DNA methylation on the wrapped length. (a) Frequency distribution of the wrapped length of unmethylated DNA around the histone octamer. (b) Frequency distribution of the wrapped length of methylated DNA around the histone octamer. A Gaussian-fitted curve is overlaid on each histogram.

(a)
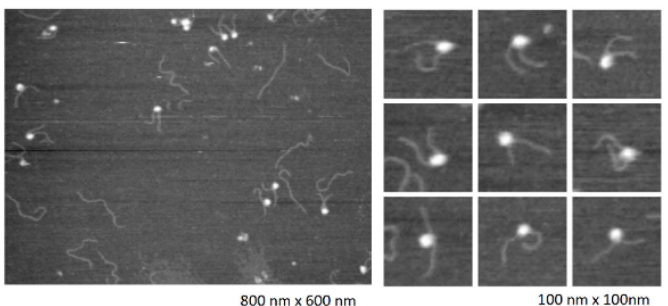

(b)
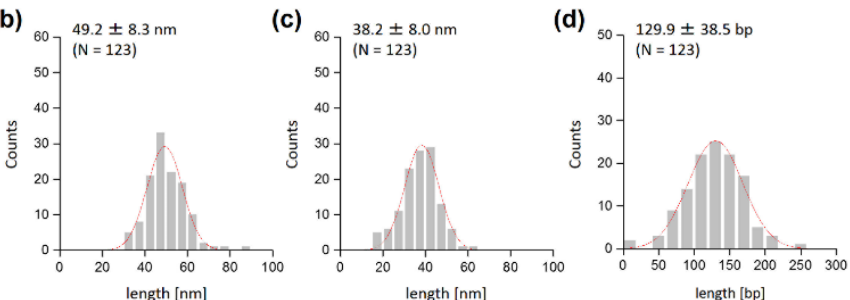

Fig. 5 AFM imaging of the cytosine-hydroxymethylated nucleosome. (a) AFM images of nucleosomes that were reconstituted on hydroxymethylated DNA. Lower magnification: image size, $800 \mathrm{~nm} \times 600 \mathrm{~nm}$; higher magnification: image size, $100 \mathrm{~nm} \times 100 \mathrm{~nm}$. (b) Histogram of the measured contour lengths of the longer arms. (c) Histogram of the measured contour lengths of the shorter arms. (d) Frequency distribution of the wrapped length 
of methylated DNA around the histone octamer. A Gaussian-fitted curve is overlaid on each histogram.

In addition to DNA methylation, DNA demethylation is also a fundamental process for epigenetic regulation of gene expression. It has been suggested that $\mathrm{mC}$ can be demethylated via stepwise oxidization in vivo. ${ }^{12}$ Among the oxidized derivatives of $\mathrm{mC}, \mathrm{hmC}$ has been proposed as a key epigenetic mark per $s e .{ }^{27}$ Considering that the modification of DNA alters its physical properties, the reduction of hydrophobicity upon oxidization from $\mathrm{mC}$ to $\mathrm{hmC}$ should also affect the structure of nucleosomes.

To address this hypothesis, next we performed similar AFM analyses on cytosine-hydroxymethylated nucleosomes. The results obtained are shown in Fig. 5. The mean lengths of the two arms were $49.2 \pm 8.3 \mathrm{~nm}$ and $38.2 \pm 8.0 \mathrm{~nm}$, respectively. These values were larger than the values obtained for the methylated nucleosomes, but smaller than those recorded for the unmethylated ones. The frequency distribution of the wrapped DNA for cytosinehydroxymethylated nucleosomes had a peak at $129.9 \pm 38.5 \mathrm{bp}$, which represented a value that was between that of unmethylated nucleosomes $(116.9 \pm 40.9 \mathrm{bp})$ and that of methylated nucleosomes $(149.7 \pm 35.5 \mathrm{bp})$. These findings indicate that the cytosinehydroxymethylated nucleosomes adopt a structural state that is different from those of both unmethylated and methylated nucleosomes. The introduction of hydroxyl groups at almost all mCs greatly reduces the hydrophobicity of DNA, which may allow the unwrapping and exposure of nucleosomal DNA on the nucleosome.

\section{Conclusions}

In the present study, we have shown that DNA methylation causes profound changes in the morphology of nucleosomes, with increase in the wrapping of DNA around the histone octamer. This overwrapping was mitigated when the mCs in the template DNA were substituted with hmCs. However, the extent of wrapping in cytosine-hydroxymethylated nucleosomes was still larger than that of unmodified nucleosomes. These findings suggest that three different types of DNA modification yield different wrapping states of nucleosomes, probably reflecting the modification-induced changes in the physical properties of DNA strands. This information regarding the interplay between DNA modifications and the morphology of nucleosomes will promote our basic understanding of the mechanisms of epigenetic regulation.

\section{Notes and references}

a Department of Chemistry, Graduate School of Science, Kyoto University, Kitashirakawa-oiwakecho, Sakyo-ku, Kyoto 606-8502, Japan

${ }^{b}$ Institute for Integrated Cell-Material Sciences (WPI-iCeMS), Kyoto University, Yoshida-ushinomiyacho, Sakyo-ku, Kyoto, 606-8501, Japan R. D. Kornberg, Science, 1974, 184, 868-871.

2 K. Luger, A. W. Mader, R. K. Richmond, D. F. Sargent and T. J. Richmond, Nature, 1997, 389, 251-260.
A. Bird, Genes. Dev., 2002, 16, 6-21.

D. Takai and P. A. Jones, Proc. Natl. Acad. Sci. U. S. A., 2002, 99, 3740-3745.

M. Esteller, Nat. Rev. Genet., 2007, 8, 286-298.

M. F. Robert, S. Morin, N. Beaulieu, F. Gauthier, I. C. Chute, A. Barsalou and A. R. MacLeod, Nat. Genet., 2003, 33, 61-65.

R. J. Klose and A. P. Bird, Trends Biochem. Sci., 2006, 31, 89-97.

S. C. Wu and Y. Zhang, Nat. Rev. Mol. Cell Biol., 2010, 11, 607-620.

Y. Kawasaki, J. Lee, A. Matsuzawa, T. Kohda, T. KanekoIshino and F. Ishino, Sci. Rep., 2014, 4, 3658.

M. Tahiliani, K. P. Koh, Y. Shen, W. A. Pastor, H. Bandukwala, Y. Brudno, S. Agarwal, L. M. Iyer, D. R. Liu, L. Aravind and A. Rao, Science, 2009, 324, 930-935.

Y. F. He, B. Z. Li, Z. Li, P. Liu, Y. Wang, Q. Tang, J. Ding, Y. Jia, Z. Chen, L. Li, Y. Sun, X. Li, Q. Dai, C. X. Song, K. Zhang, C. He and G. L. Xu, Science, 2011, 333, 1303-1307.

S. Ito, L. Shen, Q. Dai, S. C. Wu, L. B. Collins, J. A. Swenberg, C. He and Y. Zhang, Science, 2011, 333, 13001303.

H. Wu and Y. Zhang, Cell, 2014, 156, 45-68.

L. S. Shlyakhtenko, A. Y. Lushnikov and Y. L. Lyubchenko, Biochemistry, 2009, 48, 7842-7848.

N. A. Filenko, C. Kolar, J. T. West, S. A. Smith, Y. I. Hassan, G. E. O. Borgstahl, J. Zempleni, and Y. L. Lyubchenko, PLoS One, 2011, 6, e16299.

P. T. Lowary and J. Widom, J. Mol. Biol., 1998, 276, 1942.

K. Hizume, S. H. Yoshimura, H. Maruyama, J. Kim, H. Wada and K. Takeyasu, Arch. Histol. Cytol., 2002, 65, 405-413.

K. Hizume, S. H. Yoshimura and K. Takeyasu, Biochemistry, 2005, 44, 12978-12989.

K. Ura, J. J. Hayes and A. P. Wolffe, EMBO J., 1995, 14, 3752-3765.

K. Ura and A. P. Wolffe, Methods. Enzymol., 1996, 274, 257-271. 
21 B. D. Brower-Toland, C. L. Smith, R. C. Yeh, J. T. Lis, C. L. Peterson and M. D. Wang, Proc. Natl. Acad. Sci. U. S. A., 2002, 99, 1960-1965.

S. Mihardja, A. J. Spakowitz, Y. Zhang and C. Bustamante, Proc. Natl. Acad. Sci. U. S. A., 2006, 103, 15871-15876.

G. Li, M. Levitus, C. Bustamante and J. Widom, Nat. Struct. Mol. Biol., 2005, 12, 46-53.

H. S. Tims and J. Widom, Methods, 2007, 41, 296-303.

J. S. Choy, S. Wei, J. Y. Lee, S. Tan, S. Chu and T. H. Lee, J. Am. Chem. Soc., 2010, 132, 1782-1783.

26 J. Y. Lee and T. H. Lee, J. Am. Chem. Soc., 2012, 134, 173-175.

L. Shen and Y. Zhang, Curr. Opin. Cell Biol., 2013, 25, 289-296. 